\title{
miR-135b expression downregulates Ppm1e to activate AMPK signaling and protect osteoblastic cells from dexamethasone
}

\author{
Jian-Bo Fan ${ }^{1,4, *}$, Jian-Wei Ruan ${ }^{2, *}$, Wei Liu ${ }^{1, *}$, Lun-Qing Zhu ${ }^{3}$, Xin-Hui Zhu ${ }^{1}$, Hong \\ $\mathrm{Yi}^{1}$, Sheng-Yu Cui ${ }^{1}$, Jian-Ning Zhao ${ }^{4}$, Zhi-Ming Cui ${ }^{1}$ \\ ${ }^{1}$ The Department of Orthopaedics, The Second Affiliated Hospital of Nantong University, Nantong 226001, Jiangsu, PR China \\ ${ }^{2}$ The Department of Orthopedics, The Second Affiliated Hospital of Soochow University, Suzhou 215000, China \\ ${ }^{3}$ The Center of Diagnosis and Treatment for Childrens' Bone Disease, Childrens' Hospital Affiliated to Soochow University, \\ Suzhou 215000, Jiangsu, PR China \\ ${ }^{4}$ Department of Orthopaedics, Jinling Hospital, Nanjing Medical University, Nanjing 210008, Jiangsu, PR China \\ *These authors have contributed equally to this work \\ Correspondence to: Zhi-Ming Cui, email: zhimingcuiorth@163.com \\ Keywords: dexamethasone (Dex), osteoblastic cells, microRNA-135b, AMP-activated protein kinase (AMPK), phosphatase 1E \\ (Ppmle)
}

Received: July 07, $2016 \quad$ Accepted: September 06, $2016 \quad$ Published: September 20, 2016

\section{ABSTRACT}

Activation of AMP-activated protein kinase (AMPK) could potently protect osteoblasts/osteoblastic cells from dexamethasone (Dex). We aim to induce AMPK activation via microRNA ("miRNA") downregulation of its phosphatase Ppm1e. We discovered that microRNA-135b ("miR-135b") targets the 3' untranslated regions (UTRs) of Ppm1e. In human osteoblasticOB-6 cells and hFOB1.19 cells, forcedexpression of miR-135b downregulated Ppm1e and activated AMPK signaling. miR135b also protected osteoblastic cells from Dex. shRNA-induced knockdown of Ppm1e similarly activated AMPK and inhibited Dex-induced damages. Intriguingly, in the Ppm1e-silenced osteoblastic cells, miR-135b expression failed to offer further cytoprotection against Dex. Notably, AMPK knockdown (via shRNA) or dominant negative mutation abolished miR-135b-induced AMPK activation and cytoprotection against Dex. Molecularly, miR-135b, via activating AMPK, increased nicotinamide adenine dinucleotide phosphate (NADPH) activity and inhibited Dex-induced oxidative stress. At last, we found that miR-135b level was increased in human necrotic femoral head tissues, which was correlated with Ppm1e downregulation and AMPK activation. There results suggest that miR-135b expression downregulates Ppm1e to activate AMPK signaling, which protects osteoblastic cells from Dex.

\section{INTRODUCTION}

Dexamethasone (Dex) and other glucocorticoids (GC) are often prescribed to patients with inflammatory and auto-immune diseases [1]. Yet, Dex will induce cytotoxic effect to osteoblasts, which may contribute to osteoporosis and osteonecrosis [2,3]. Studies are focusing on exploring the pathological mechanisms of GC-induced osteoblast damages, and to develop possible intervention strategies [4-9]. It has also been our research focus [5, 7].

AMP-activated protein kinase (AMPK) is the master regulator of energy metabolism in mammalian cells [10]. Existing evidences have indicated that AMPK-regulated signalings are also important for cell survival, especially when cells are under certain stress conditions [11]. AMPK could activate cytoprotective autophagy to inhibit cell apoptosis $[12,13]$. Meanwhile, AMPK may also exert an anti-oxidant activity through activating nicotinamide adenine dinucleotide phosphate (NADPH) and inhibiting ATP depletion [14].

Recent studies have investigated the potential functions of AMPK in osteoblasts/osteoblastic cells. Guo et al., showed that activation of AMPK by a novel AMPK activator (Compound 13 [15]) protected osteoblasts from Dex [16]. Meanwhile, A-769662, the known AMPK activator, could offer significant protection to osteoblasts against hydrogen 
dioxide $\left(\mathrm{H}_{2} \mathrm{O}_{2}\right)$ [17]. On the other hand, inhibition of AMPK potentiated $\mathrm{H}_{2} \mathrm{O}_{2}$-induced osteoblast cell damages [18]. These results suggest that activation of AMPK likely exerts cytoprotective functions in osteoblasts/osteoblastic cells.

Phosphorylation on Thr172 of AMPK catalytic $\alpha$ subunit is vital for AMPK activation [13, 19]. Although kinase phosphorylation of this site has been extensively studied [20], the phosphatases that de-phosphorylate it are largely unknown until recently [21]. The initial data have demonstrated that protein phosphatase ce:sup $>$ / ce:sup $>/ \mathrm{Mn} 2+-$ dependent (Ppm) 1e (Ppm1e) could be a primary AMPK kinase phosphatase [21]. Ppmle, a PPM family member, was first identified as a $\mathrm{Ca}^{2+} /$ calmodulindependent protein kinase (CaMK) phosphatase. It is activated in the presence of $\mathrm{Mg}^{2+}$ and/or $\mathrm{Mn}^{2+}[22$, 23]. Ppmle is insensitive to PPP family inhibitors (i.e. calyculin A and okadaic acid) [22, 23]. Studies have shown that Ppm1e can de-phosphorylate CaMKI, nuclear CaMKIV, and CaMKII $[22,23]$.

Based on the above discussion, we would propose that inhibition of Ppm1e may activate AMPK signaling and protect osteoblasts/osteoblastic cells from Dex. Through searching miRNA databases, we found that microRNA-135b ("miR-135b") targets the 3' untranslated regions (UTRs) of Ppm1e. We showed that miR-135b expression downregulated Ppmle to activate AMPK, which protects osteoblastic cells from Dex.

\section{RESULTS}

\section{Forced expression of microRNA-135b downregulates Ppm1e but activates AMPK signaling in human osteoblastic cells}

First, through searching multiple microRNA database, we found that microRNA-135b ("miR-135b") targets the 3" untranslated regions (UTRs, Position 517-524) of human Ppmle (Figure 1A). We next established a miR-135b expressing construct (see Methods) and stably transfected it into osteoblastic cells. qRT-PCR assay results in Figure $1 \mathrm{~B}$ showed that miR-135b was indeed over-expressed in the stable OB-6 cells after transfection. Expression of miR-135b-5p was also significantly increased (Data not shown). On the other hand, Ppm1e mRNA (Figure 1C) and protein (Figure 1D) were both dramatically downregulated. Importantly, p-AMPK $\alpha$ (Thr-172) and p-acetyl-CoA carboxylase (p-ACC, Ser-79) levels were largely enhanced in the miR-135b-expresising OB-6 cells (Figure 1D), indicating AMPK signaling activation. We repeated those experiments also in osteoblastic hFOB1.19 cells. Similarly, stable hFOB1.19 cells expressing miR-135b (Figure 1E) showed depleted Ppmle (Figure 1F and 1G) but increased AMPK activation (p-AMPK $\alpha /$ p-ACC, Figure $1 G$ ). Together, these results suggest that forced expression of miR-135b decreases Ppm1e, but activates AMPK signaling in cultured human osteoblastic cells.

\section{Forced expression of microRNA-135b protects osteoblastic cells from Dex}

Recent studies have indicated that activation of AMPK could protect osteoblastic cells [16-18]. The results above demonstrated that AMPK was activated in miR-135b-expressing osteoblastic cells. We therefore wanted to know if these cells were actually protected from Dex. OB-6 osteoblastic cells were treated with Dex, which induced viability inhibition (MTT OD reduction, Figure 2A), apoptosis activation (Histone DNA ELISA OD increase, Figure 2B) and cell death (trypan blue increase, Figure 2C). Remarkably, expression of miR$135 \mathrm{~b}$ significantly attenuated above cytotoxic effects by Dex in OB-6 cells (Figure 2A-2C). In another words, OB-6 cells with miR-135b expression were resistant to Dex (Figure 2A-2C). We also repeated those experiments in hFOB1.19 cells, and similar anti-Dex effects by miR$135 \mathrm{~b}$ were noticed (Figure 2D-2F). As compared to control cells, hFOB1.19 cells expressing miR-135b were protected from Dex, showing improved viability (Figure 2D), decreased cell apoptosis (Figure 2E) and cell death (Figure 2F). These results suggest that forced expression of miR-135b indeed protects osteoblastic cells from Dex.

\section{shRNA knockdown of Ppm1e activates AMPK and protects osteoblastic cells from Dex}

We wanted to know if Ppm1e is the primary target of miR-135b in mediating its cytoprotective effect in osteoblastic cells. First, shRNA strategy was applied to stably knockdown Ppm1e. qRT-PCR results in Figure 3A and Western blot results in Figure 3B confirmed Ppm1e knockdown by the targeted shRNAs in OB-6 cells. Notably, two shRNAs ("1/2") targeting non-overlapping sequences of human Ppmle were applied, and each of them efficiently downregulated Ppmle (Figure 3A and 3B). On the other hand, AMPK activation, evidenced by p-AMPK/p-ACC, was increased in Ppm1e-silneced OB-6 cells (Figure 3B). Significantly, Dex-induced OB-6 cell viability reduction (Figure $3 \mathrm{C}$ ) and apoptosis activation (Figure 3D) were dramatically attenuated in Ppm1eknockdown cells. The Ppmle shRNA experiments were also repeated in hFOB1.19 cells, and similar results were obtained (Data not shown).Therefore, Ppmle silence activates AMPK and protects osteoblastic cells from Dex.

We also expressed miR-135b in the Ppm1e-silenced OB-6 cells. qRT-PCR results in Figure 3E confirmed miR135b over-expression in Ppm1e-shRNA ("-1")-containing OB-6 cells. Ppmle mRNA level was again decreased in these cells (Figure 3F). Significantly, expression of miR$135 \mathrm{~b}$ failed to further offer cytoprotection against Dex in the Ppm1e-silenced cells (Figure 3G and 3H). These results suggest that Ppmle is likely the primary target of miR-135b in mediating its cytoprotective effect in osteoblastic cells. 


\section{AMPK knockdown or mutation abolishes miR- 135b-induced cytoprotection in osteoblastic cells}

Next, we wanted to know if AMPK activation was the reason of miR-135b-meidated cytoprotection in osteoblastic cells. Genetic strategies were applied to inhibit AMPK activation. AMPK $\alpha$ shRNA or a dominant negative AMPK $\alpha$ (dn-AMPK $\alpha$, T172A) was introduced to the miR-135b-expressing OB-6 cells. Western blot results in Figure 4A confirmed the phenotypes (AMPK $\alpha$ knockdown or mutation) of these cells. Expectably, miR$135 \mathrm{~b}$ or Ppm1e mRNA expression was not changed in these cells (Figure 4B). Importantly, AMPK knockdown or dominant negative mutation almost blocked AMPK activation (p-AMPK/p-ACC) in miR-135b-expressing OB-6 cells (Figure 4A). Consequently, miR-135b-induced cytoprotection against Dex was almost nullified in OB-6 cells (Figure 4C and 4D). In another words, miR-135b was no longer cytoprotective in AMPK-depleted or AMPK-mutated cells. Similar in hFOB1.19 cells, AMPKa shRNA or dominant negative mutation largely attenuated miR-135b-induced cytoprotection against Dex (Figure 4E and $4 \mathrm{~F}$ ). Based on these results, we conclude that AMPK activation mediates miR-135b-induced cytoprotection against Dex in osteoblastic cells.

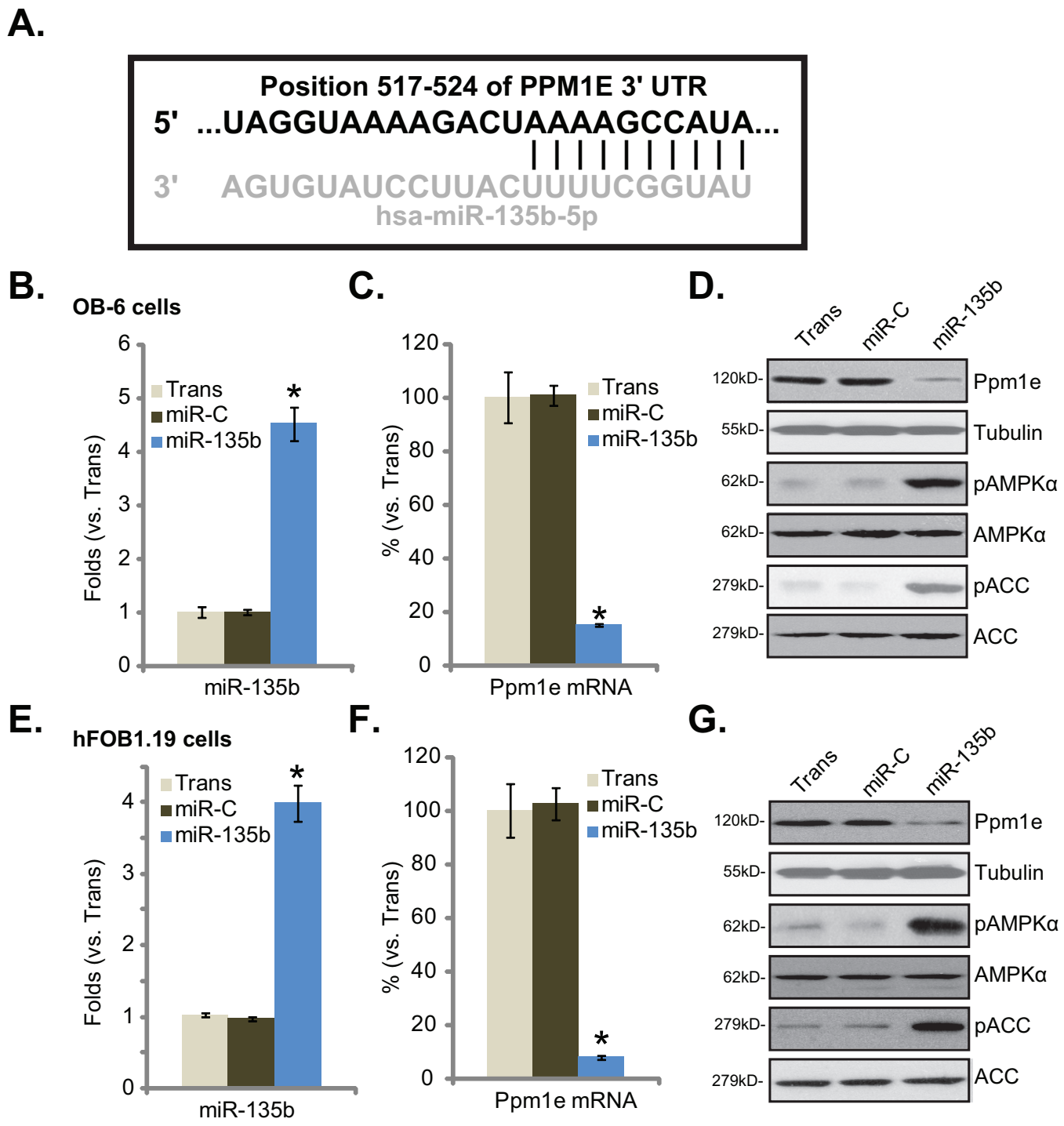

Figure 1: Forced expression of microRNA-135b downregulates Ppm1e but activates AMPK signaling in human osteoblastic cells. A. microRNA-135b ("miR-135b") targets the 3" untranslated regions (UTRs, position 517-524) of human Ppmle (Figure 1A). Human osteoblastic OB-6 cells B-D. or hFOB1.19 cells E-G. were transfected with microRNA-135b ("miR-135b") or nonsense control microRNA ("miR-C"), and stable cells were established. Expressions of miR-135b (B and E) and Ppmle mRNA (C and F) were tested by quantitative real-time PCR ("qRT-PCR") assay; Expression of listed proteins in these cells were tested by Western blot assay (D and G). Experiments in this figure were repeated four times, and similar results were obtained. "Trans" stands for transfection reagents only (B-G). * $p<0.05$ vs. group "miR-C" (B, C, E and F). 
A.

OB-6 cells

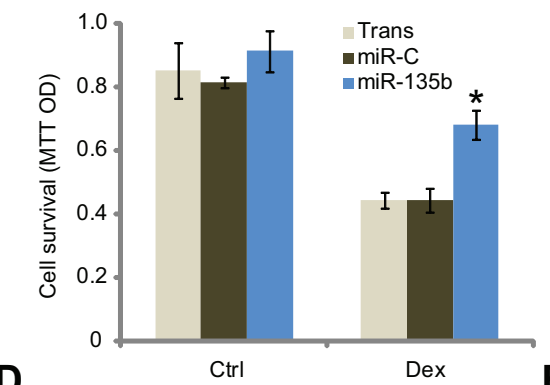

D.

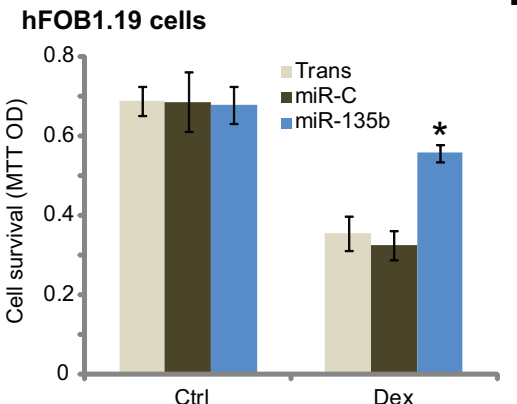

B.
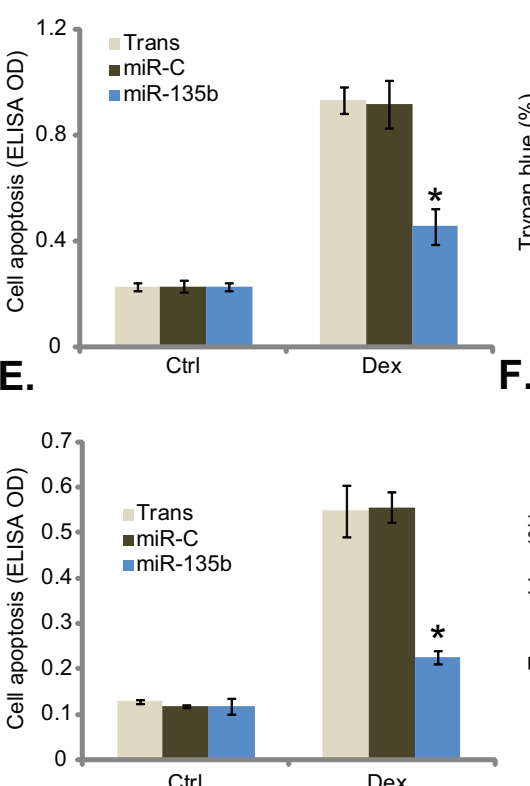

C.

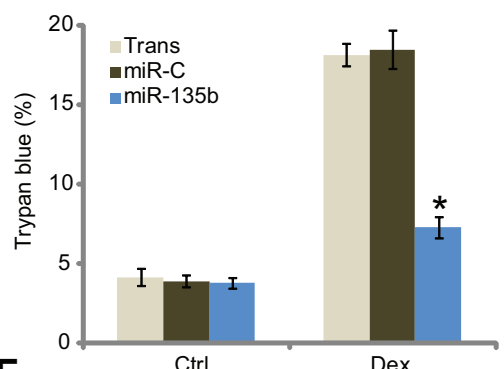

F.

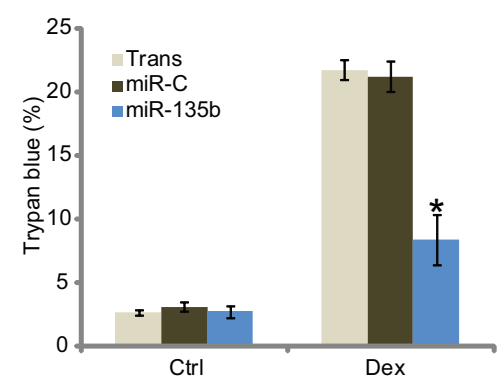

Figure 2: Forced expression of microRNA-135b protects osteoblastic cells from Dex. Stable osteoblastic OB-6 cells A-C. or hFOB1.19 cells D-F. expressing microRNA-135b ("miR-135b") or non-sense control microRNA ("miR-C") were treated with or without Dex $(1 \mu \mathrm{M})$ for 24 hours, cell viability (MTT assay, A and D), apoptosis (Histone DNA ELISA assay, B and E) and cell death (trypan blue assay, C and F) were tested. Experiments in this figure were repeated four times, and similar results were obtained. "Ctrl" stands for untreated control group. "Trans" stands for transfection reagents only. * $p<0.05$ vs. "miR-C" cells with Dex treatment.

A.

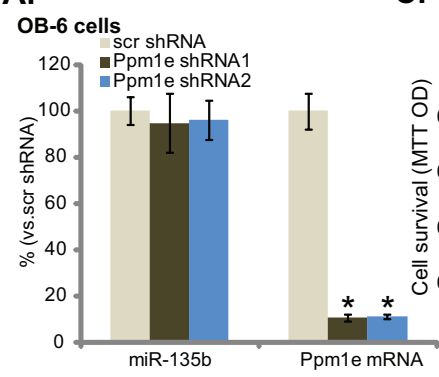

B.

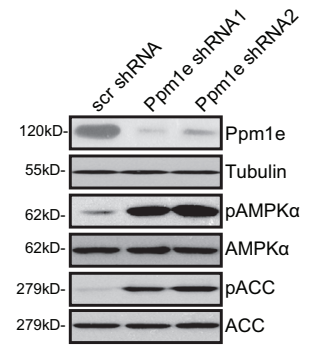

C.

D.

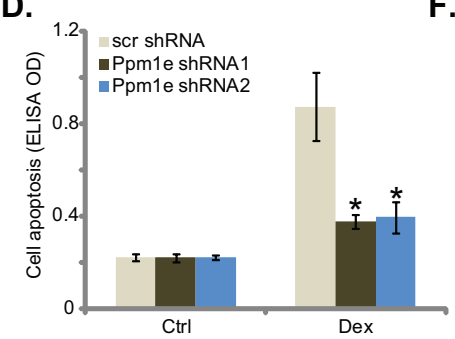

E.

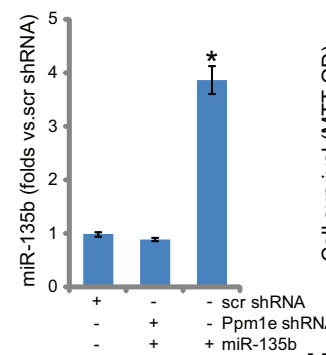

F.

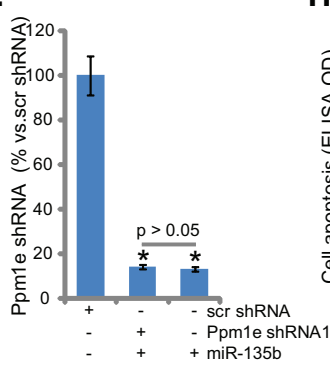

G.

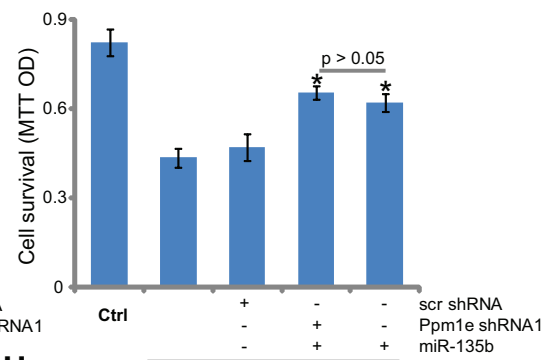

H.

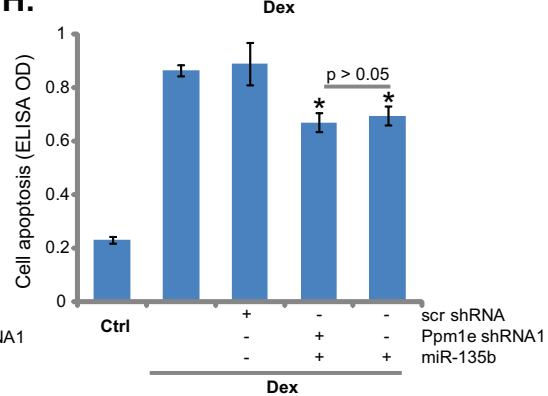

Figure 3: shRNA knockdown of Ppm1e activates AMPK and protects osteoblastic cells from Dex. OB-6 cells were infected with lentiviral Ppmle shRNA ("-1 or -2") or non-sense control shRNA ("scr shRNA"), and stable cells were established. miR-135b and Ppmle mRNA levels were tested (A, qRT-PCR assay), Ppmle protein expression and AMPK activation (p-AMPK/p-ACC) were also tested (B, Western blot assay). Above cells were treated with or without Dex $(1 \mu \mathrm{M})$ for 24 hours, cell viability (C, MTT assay) and apoptosis (D, Histone DNA ELISA assay) were shown. Ppm1e shRNA (“-1”)-expressing OB-6 cells were transfected with miR-135b expressing construct, expressions of miR-135b E. and Ppmle mRNA F. were tested by qRT-PCR assay, these cells were also treated with or without $\operatorname{Dex}(1 \mu \mathrm{M})$ for 24 hours, cell viability G. and apoptosis H. were shown. "Ctrl" stands for untreated control group. Experiments in this figure were repeated four times, and similar results were obtained. * $p<0.05$ vs. "scr shRNA" cells (A, C-H). 
A.

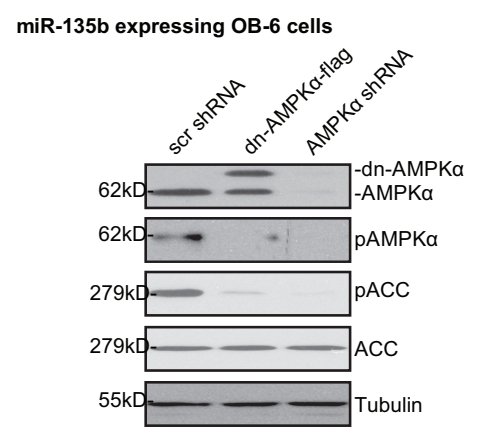

C.

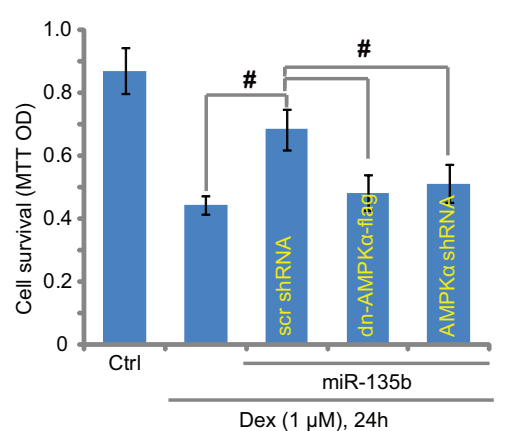

E.

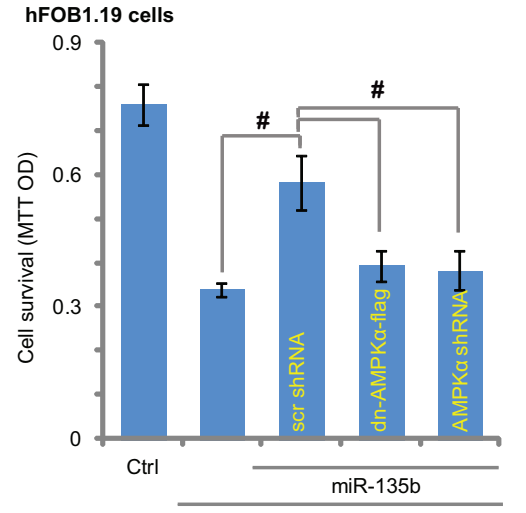

G.

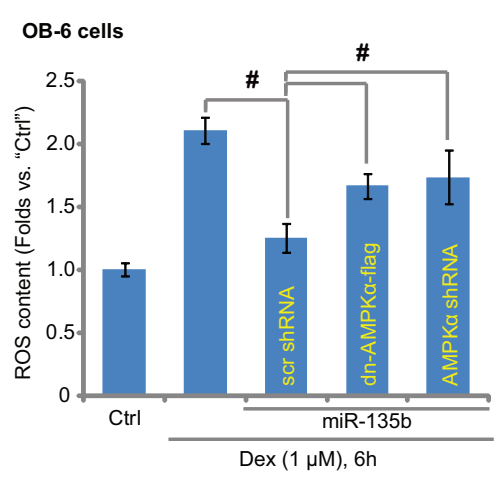

B.

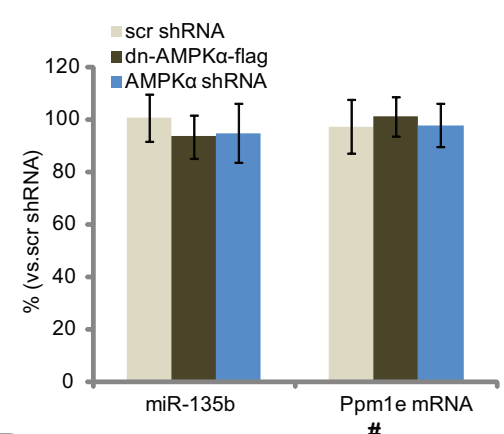

D.

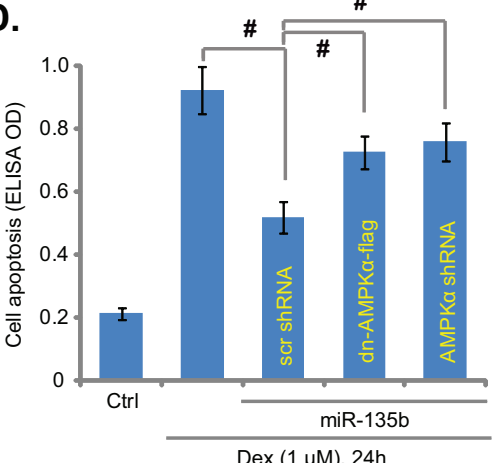

F.

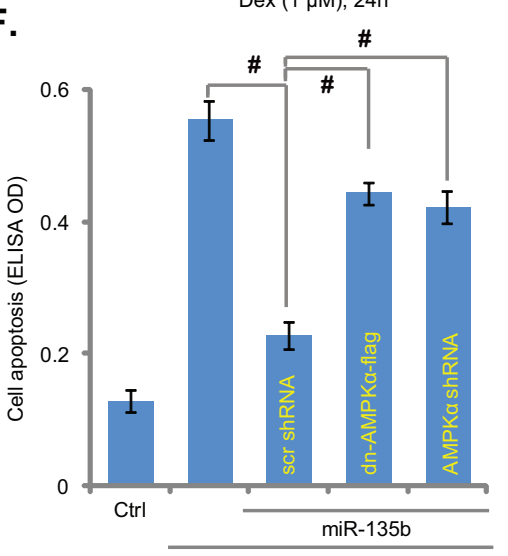

H.

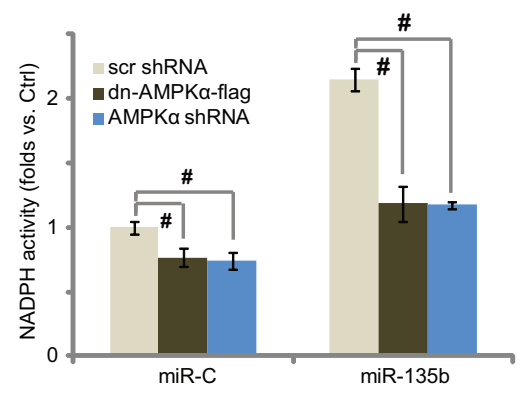

Figure 4: AMPK knockdown or mutation abolishes miR-135b-induced cytoprotection in osteoblastic cells. miR-135b expressing OB-6/hFOB1.19 cells cells were constructed with AMPK $\alpha$ shRNA, dominant negative AMPK $\alpha$ ("dn-AMPK $\alpha$-flag", T172A) or the scramble control shRNA ("Scr shRNA"), expressions of listed proteins in these cells were tested by Western blots (A, for OB-6 cells); miR-135b and Ppmle mRNA expressions were also tested (B, qRT-PCR assay, for OB-6 cells). Above cells were treated with or without Dex $(1 \mu \mathrm{M})$ for indicated periods of time, cell viability (MTT assay, $\mathbf{C}$ and $\mathbf{E}$ ), apoptosis intensity (Histone DNA ELISA assay, D and $\mathbf{F}$ ) and ROS content (DCFH-DA fluorescent dye assay, $\mathbf{G}$ ) were tested. NADPH activity in above cells was also shown $\mathbf{H}$. Experiments in this figure were repeated four times, and similar results were obtained. "Ctrl" stands for untreated control group. ${ }^{*} p<0.05$. 
Recent studies have shown that AMPK activation inhibits Dex-induced ROS production, therefore protecting osteoblasts/osteoblastic cells [16]. We also noticed a significant ROS increase in Dex-treated OB-6 cells (Figure 4G). Such an effect by Dex was largely inhibited by expression of miR-135b (Figure 4G). Significantly, AMPK knockdown or dominant negative mutation almost completely abolished miR-135b-induced anti-oxidant ability (Figure 4G). It has been suggested that AMPK exerts anti-oxidant activity via activating NADPH $[14,16$, $18,24]$. Here, we found that miR-135b-induced NADPH activity increase required AMPK activation (Figure 4H). AMPK shRNA knockdown or dominant negative mutation almost completely blocked NADPH activity increase by miR-135b (Figure 4H). The similar results were also obtained in hFOB1.19 cells (Data not shown). Thus, miR135b activates AMPK-NADPH signaling to possibly inhibit Dex-induced oxidative stress and osteoblastic cell death.

\section{Upregulation of miR-135b in patients' osteonecrosis tissues}

A recent study by She et al., showed that, as compared to the normal femoral head tissues, AMPK activation was increased in patients' necrotic femoral head tissues [18]. Here, we also observed AMPK activation (p-ACC intensity increase) in the necrotic femoral head tissues (vs. the surrounding normal femoral head tissues) (Figure 5A). Interestingly, in the necrotic tissues, the level of miR-135b was also increased (Figure 5B), and the Ppmle (protein and mRNA) level was decreased (Figure 5C and 5D). These results indicate that miR-135b upregulation and Ppmle depletion could be the reason of AMPK activation in patients' necrotic femoral head tissues.

\section{DISCUSSIONS AND CONCLUSIONS}

Here we showed that miR-135b selectively targets the AMPK phosphatase Ppmle [21], whose expression induced a profound AMPK activation in osteoblastic cells. Significantly, miR-135b expression or Ppmle shRNA knockdown, which also activated AMPK signalings, significantly protected osteoblastic cells from Dex. Intriguingly, after Ppm1e was silenced, miR-135b was not able to further protect osteoblastic cells from Dex. Therefore, Ppm1e could be the primary target of miR$135 \mathrm{~b}$ in mediating its cytoprotective effect. Additionally, we showed that AMPK knockdown or mutation abolished miR-135b-induced cytoprotection against Dex in osteoblastic cells, supporting a critical function of AMPK activation in miR-135b's actions in osteoblastic cells.

Dex treatment is shown to induce oxidative stress in osteoblasts/osteoblastic cells, which subsequently contributes to cell death and apoptosis $[16,25]$. On the other hand, ROS suppression could inhibit Dex-induced osteoblastic cell damages [16]. Interestingly, recent studies have proposed potent anti-oxidant activity of AMPK $[14,16,18,26]$. AMPK is shown to participate in maintaining NADPH content [14]. AMPK phosphorylates and inhibits ACC, thus decreasing NADPH consumption [14]. Meanwhile, AMPK activation was shown to induce NADPH synthesis by fatty-acid oxidation [14]. A very recent study by Guo et al., showed that compound 13 (C13), an $\alpha 1$-selective AMPK activator, activated NADPH signaling to inhibit Dex-induced ROS production, therefore protecting osteoblastic cells from Dex [16]. Here we showed that miR-135b expression increased NADPH activity and inhibited Dex-induced oxidative stress in osteoblastic cells, and such effects were almost reversed by AMPK knockdown or mutation. Therefore, we suggest
A.

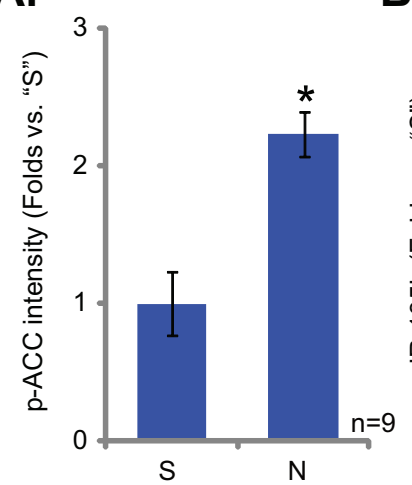

N. Necrotic femoral head tissues

S. Surrounding normal femoral head tissues
B.

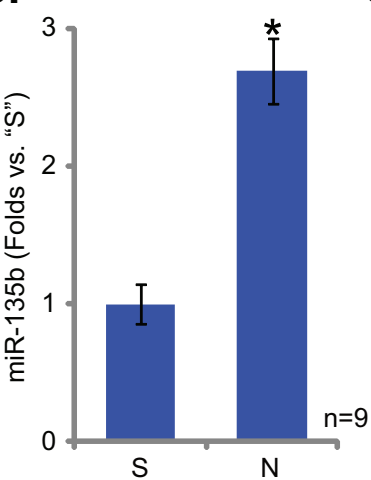

C

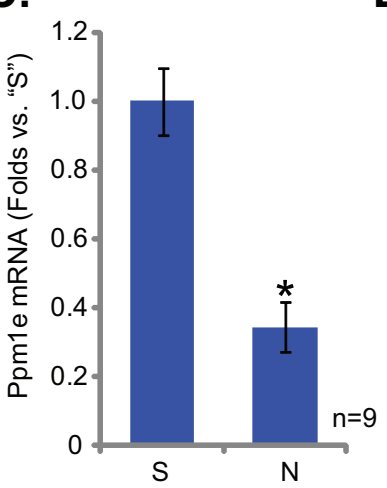

D.

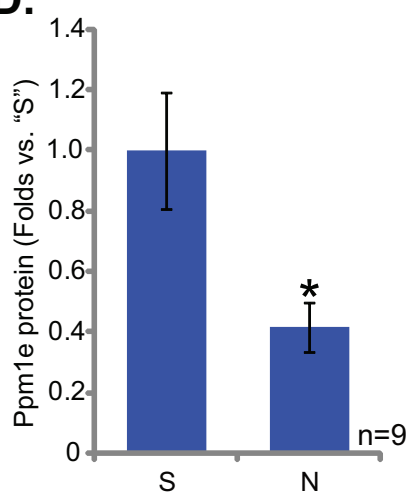

Figure 5: Upregulation of miR-135b in patients' osteonecrosis tissues. Western blots analyzing the expression of listed proteins in surgery isolated femoral head tissues (both normal and necrotic) from GC-taking patients; ACC phosphorylation A. and Ppm1e protein (D, normalized to Tubulin) were quantified. Expressions of miR-135b B. and Ppm1e mRNA C. were also shown (qRT-PCR assay) in above tissues. * $p<0.05$ vs. "S" tissues. 
that miR-135b activates AMPK to exert anti-oxidant activity and to protect osteoblastic cells from Dex.

Prolonged and/or overdose GC usage would cause secondary osteoporosis [27, 28] and/or osteonecrosis [29]. Reduced number of osteoblasts, suppressed osteoblastogenesis and increased osteoblast cell apoptosis were observed in the bones of the GC-taking patients [27, 28]. Here we found that miR-135b attenuated Dex-induced osteoblastic cell death and apoptosis. Significantly, miR$135 \mathrm{~b}$ level was increased in human necrotic femoral head tissues, which was correlated with Ppmle downregulation and AMPK activation. Thus, it will be interesting to test the possible effect of miR-135b against GC-induced osteoporosis and/or osteonecrosis in vivo. In summary, our preclinical results indicate that miR-135b expression downregulates Ppm1e to activate AMPK signaling and protect osteoblastic cells from Dex.

\section{MATERIALS AND METHODS}

\section{Chemicals and reagents}

Dex was purchased from Sigma (Shanghai, China). All cell culture reagents were obtained from Gibco (Shanghai, China). Antibodies of p-AMPK $\alpha 1$ (Thr172,

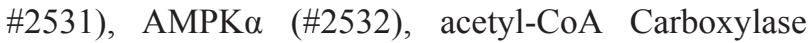
(ACC, \#3662), p-ACC (Ser79, \#3661) and ( $\beta$-) Tubulin (\#2146) were purchased from were obtained from Cell Signaling Technology (Beverly, MA). Ppmle antibody was purchased from Abnova (\#PAB21197, Taibei, China).

\section{Cell culture}

The OB-6 [2] and hFOB1.19 [30] human osteoblastic cells were purchased from the Cell Bank of Shanghai Institute of Biological Science (Shanghai, China), and were cultured as described $[2,30]$.

\section{Quantitative real-time polymerase chain reaction (qRT-PCR) assay}

Total RNA was extracted with the SV total RNA purification system (Promega, Shanghai, China). RNA was then reverse-transcribed through the reverse transcriptase (Promega, Madison, WI, USA). cDNA derived from 1.0 $\mu \mathrm{g}$ of total RNA was amplified by quantitative real-time polymerase chain reaction ("qRT-PCR"). The SYBR Green PCR kit (Applied Biosystems) was then utilized to detect expression of listed mRNAs. GAPDH primers were described in our previous studies [31]. Human Ppmle primers were described in previous studies [32]. PCR was performed in triplicate and was conducted using a Real-Time PCR Detection System (7500; ABI, Shanghai, China). mRNA expression was quantified using the ${ }^{{ }^{\Delta}} \mathrm{Ct}$ method. GAPDH served as the internal control. For miRNA analysis, real-time PCR was performed using PrimeScript miRNA RT-PCR Kit (Takara, Tokyo, Japan) according to the manufacturer's protocols. The miR-135b primers were described early [33, 34]. All the primers and sequences were synthesized by Genepharm (Shanghai, China).

\section{Forced miR-135b expression}

Pre-miR-135b (see its sequence in studies [33, 34]) was sub-cloned into pSuper-neo (OligoEngine, Seattle, WA) to generate miR-135b expression vector. Osteoblastic cells were seeded onto 6-well plates with $50-60 \%$ confluence. The miR $-135 \mathrm{~b}$ construct $(0.10 \mu \mathrm{g} /$ $\mathrm{mL}$ of each well) was transfected to the osteoblastic cells via Lipofectamine 2000 protocol (Invitrogen, Shanghai, China). After 24 hours, cells were subjected to neomycin $(1.0 \mu \mathrm{g} / \mathrm{mL})$ selection for $10-12$ days. miR-135b expression in the resulting stable cells was tested by the qRT-PCR assay. Control cells were transfected with nonsense scramble microRNA-control ("miR-C") (a gift from Dr. Lu's group [35]).

\section{Western blot assay}

As described in our previous studies [5,7], cell lysates were extracted via RIPA lysis buffer (Bio-sky, Nanjing, China). Protein concentration was determined, and aliquots of $30 \mu \mathrm{g}$ lysates per sample were electrotransferred on $10-12 \%$ SDS-PAGE gel, following by transfer to PVDF membranes. The blots were then incubated with designated primary antibodies and appropriate secondary antibodies. The antigen-antibody binding was detected via enhanced chemiluminescence (ECL) reagents.

\section{Cell death detection}

Osteoblastic cells were seeded onto 12-well plates. Following the indicated treatment, cells were trypsinized. Cell death percentage was determined by counting cells using a cytometer after addition of trypan blue, which stained the cytoplasm of dead cells. Cell death percentage $(\%)=$ the number of trypan blue stained cells/the number of total cells $(\times 100 \%)[7]$.

\section{Cell viability assay}

Cell viability was measured via the routine 3-[4,5-dimethylthylthiazol-2-yl]-2,5 diphenyltetrazolium bromide (MTT) assay described in our previous studies $[5,7]$.

\section{Apoptosis assay by enzyme-linked immunosorbent assay (ELISA)}

As described [31], we applied the Histone-DNA ELISA Detection Kit (Roche, Palo Alto, CA) to quantify cell apoptosis following indicated treatments. 


\section{shRNA knock and stable cell selection}

The two lentiviral shRNAs against human Ppmle were designed, synthesized and verified by Genepharm (Shanghai, China). The AMPK $\alpha$ shRNA was described in our previous study [31]. Osteoblastic cells were seeded onto 6-well plates with $50 \%$ of confluence. The lentiviral shRNA $(10 \mu \mathrm{L} / \mathrm{mL})$ were added directly to the cells for 24 hours. Afterwards, cells were further cultured in puromycin $(1 \mu \mathrm{g} / \mathrm{mL})$-containing medium, until resistant colonies can be identified (2-3 weeks). The expression of target protein (AMPK $\alpha$ or Ppm1e) in stable cells was tested by Western blot assay. Same amount of scramble non-senesce lentiviral shRNA (Santa Cruz) was added to the control cells.

\section{AMPK dominant negative mutation}

The dominant negative mutant of AMPK $\alpha$ (dnAMPK $\alpha$, T172A) construct was a gift from Dr. Lu's group at Nanjing Medical University [36]. Osteoblastic cells were seeded onto 6-well plates with $50 \%$ of confluence. dn-AMPK $\alpha$ cDNA $(0.10 \mu \mathrm{g} / \mathrm{mL})$ was transfected to osteoblastic cells via the Lipofectamine 2000 protocol [36], and stable cells were selected via neomycin $(2 \mu \mathrm{g} /$ $\mathrm{mL}$, Sigma). Transfection efficiency was always verified via Western blot assay in the stable cells.

\section{NADPH activity assay}

NADPH activity assay was described in our and other studies $[18,37]$. Briefly, after treatment of cells, the lysates were incubated with NADP-cycling buffer plus glucose-6-phosphate dehydrogenase (G6PD, Sigma) at $60^{\circ} \mathrm{C}$ for $30 \mathrm{~min}$ [18]. Afterwards, glucose 6-phosphate (G6P, Sigma) was added to the mixture, and the change in absorbance at $570 \mathrm{~nm}$ was measured every $30 \mathrm{~s}$ for 4 min at $30^{\circ} \mathrm{C}$. The concentration of NADP+ was calculated by subtracting [NADPH] from [total NADP]. NADPH activity was then calculated through NADPH/ NADP+ [37].

\section{Reactive oxygen species (ROS) measurement}

ROS production was measured via a DCFH-DA fluorescent dye (Invitrogen). Briefly, following the applied treatment, cells were incubated with $1 \mu \mathrm{M}$ of DCFH-DA at $37^{\circ} \mathrm{C}$ for $30 \mathrm{~min}$. Cells were then washed and analyzed for fluorescence using the flow cytometer (BD Biosciences, Shanghai, China). The ROS level in the treatment group was normalized to that of control group.

\section{Human tissue collection and analysis}

Surgery-isolated fresh necrotic femoral head tissues and their surrounding normal femoral head tissues were collected from Dex-taking patients. Fresh tissue specimens were dissolved in tissue lysis buffer (BiYunTian Biotechnology Research Institute, Nantong, China) and were subjected to qRT-PCR assay and Western blot assay. The clinical examinations were approved by the institutional review board and ethics committee of all authors' institutions, and written informed consent was obtained from each patient. A total of 9 patients were included. All clinical investigations were conducted according to the principles expressed in the Declaration of Helsinki.

\section{Statistics}

The data presented were mean \pm standard deviation (SD). Statistical differences were analyzed by one-way ANOVA followed by multiple comparisons performed with post hoc Bonferroni test (SPSS version 18.0). Values of $p<0.05$ were considered statistically significant. The significance of any differences between two groups was evaluated via the paired-samples $t$ test (Excel, 2007) when appropriated.

\section{ACKNOWLEDGMENTS}

This work was generously supported by grants from the National Natural Science Foundation of China (81501866), and the Science and Technology Program of Nantong (MS22015051). The funders had no role in study design, data collection and analysis, decision to publish, or preparation of the manuscript.

\section{CONFLICTS OF INTEREST}

No conflict of interests were stated.

\section{REFERENCES}

1. Schacke H, Docke WD, Asadullah K. Mechanisms involved in the side effects of glucocorticoids. Pharmacol Ther. 2002; 96:23-43.

2. Ding H, Wang T, Xu D, Cha B, Liu J, Li Y. Dexamethasoneinduced apoptosis of osteocytic and osteoblastic cells is mediated by TAK1 activation. Biochem Biophys Res Commun. 2015; 460:157-163.

3. Yun SI, Yoon HY, Jeong SY, Chung YS. Glucocorticoid induces apoptosis of osteoblast cells through the activation of glycogen synthase kinase 3 beta. J Bone Miner Metab. 2009; 27:140-148.

4. Ji F, Mao L, Liu Y, Cao X, Xie Y, Wang S, Fei H. K6PC-5, a novel sphingosine kinase 1 (SphK1) activator, alleviates dexamethasone-induced damages to osteoblasts through activating SphK1-Akt signaling. Biochem Biophys Res Commun. 2015; 458:568-575.

5. Fan JB, Liu W, Zhu XH, Yuan K, Xu DW, Chen JJ, Cui ZM. EGFR-AKT-mTOR activation mediates epiregulin-induced 
pleiotropic functions in cultured osteoblasts. Mol Cell Biochem. 2015; 398:105-113.

6. Zhen YF, Wang GD, Zhu LQ, Tan SP, Zhang FY, Zhou XZ, Wang XD. P53 dependent mitochondrial permeability transition pore opening is required for dexamethasoneinduced death of osteoblasts. J Cell Physiol. 2014; 229:1475-1483.

7. Fan JB, Liu W, Yuan K, Zhu XH, Xu DW, Chen JJ, Cui ZM. EGFR trans-activation mediates pleiotrophininduced activation of Akt and Erk in cultured osteoblasts. Biochem Biophys Res Commun. 2014; 447: 425-430.

8. Plotkin LI, Weinstein RS, Parfitt AM, Roberson PK, Manolagas SC, Bellido T. Prevention of osteocyte and osteoblast apoptosis by bisphosphonates and calcitonin. J Clin Invest. 1999; 104:1363-1374.

9. Gohel A, McCarthy MB, Gronowicz G. Estrogen prevents glucocorticoid-induced apoptosis in osteoblasts in vivo and in vitro. Endocrinology. 1999; 140:5339-5347.

10. Carling D, Thornton C, Woods A, Sanders MJ. AMPactivated protein kinase: new regulation, new roles? Biochem J. 2012; 445:11-27.

11. Wang S, Song P, Zou MH. AMP-activated protein kinase, stress responses and cardiovascular diseases. Clin Sci (Lond). 2012; 122:555-573

12. Kim I, He YY. Targeting the AMP-Activated Protein Kinase for Cancer Prevention and Therapy. Front Oncol. 2013; $3: 175$.

13. Mihaylova MM, Shaw RJ. The AMPK signalling pathway coordinates cell growth, autophagy and metabolism. Nat Cell Biol. 2011; 13:1016-1023.

14. Jeon SM, Chandel NS, Hay N. AMPK regulates NADPH homeostasis to promote tumour cell survival during energy stress. Nature. 2012; 485:661-665.

15. Hunter RW, Foretz M, Bultot L, Fullerton MD, Deak M, Ross FA, Hawley SA, Shpiro N, Viollet B, Barron D, Kemp BE, Steinberg GR, Hardie DG, Sakamoto K. Mechanism of action of compound-13: an alpha1selective small molecule activator of AMPK. Chem Biol. 2014; 21:866-879.

16. Guo S, Mao L, Ji F, Wang S, Xie Y, Fei H, Wang XD. Activating AMP-activated protein kinase by an alpha1 selective activator Compound 13 attenuates dexamethasoneinduced osteoblast cell death. Biochem Biophys Res Commun. 2016.

17. Zhu Y, Zhou J, Ao R, Yu B. A-769662 protects osteoblasts from hydrogen dioxide-induced apoptosis through activating of AMP-activated protein kinase (AMPK). Int J Mol Sci. 2014; 15:11190-11203.

18. She C, Zhu LQ, Zhen YF, Wang XD, Dong QR. Activation of AMPK protects against hydrogen peroxide-induced osteoblast apoptosis through autophagy induction and NADPH maintenance: New implications for osteonecrosis treatment? Cell Signal. 2014; 26:1-8.
19. Hardie DG, Ross FA, Hawley SA. AMPK: a nutrient and energy sensor that maintains energy homeostasis. Nat Rev Mol Cell Biol. 2012; 13:251-262.

20. Hardie DG. AMP-activated/SNF1 protein kinases: conserved guardians of cellular energy. Nat Rev Mol Cell Biol. 2007; 8:774-785.

21. Voss M, Paterson J, Kelsall IR, Martin-Granados C, Hastie CJ, Peggie MW, Cohen PT. Ppm1E is an in cellulo AMPactivated protein kinase phosphatase. Cell Signal. 2011; 23:114-124.

22. Takeuchi M, Taniguchi T, Fujisawa H. Identification and characterization of nuclear localization signals of CaMKP-N. J Biochem. 2004; 136:183-188.

23. Takeuchi M, Ishida A, Kameshita I, Kitani T, Okuno $\mathrm{S}$, Fujisawa $\mathrm{H}$. Identification and characterization of CaMKP-N, nuclear calmodulin-dependent protein kinase phosphatase. J Biochem. 2001; 130:833-840.

24. Balteau M, Van Steenbergen A, Timmermans AD, Dessy C, Behets-Wydemans G, Tajeddine N, Castanares-Zapatero D, Gilon P, Vanoverschelde JL, Horman S, Hue L, Bertrand L, Beauloye C. AMPK activation by glucagon-like peptide-1 prevents NADPH oxidase activation induced by hyperglycemia in adult cardiomyocytes. Am J Physiol Heart Circ Physiol. 2014.

25. Almeida M, Han L, Ambrogini E, Weinstein RS, Manolagas SC. Glucocorticoids and tumor necrosis factor alpha increase oxidative stress and suppress Wnt protein signaling in osteoblasts. J Biol Chem. 2011; 286:44326-44335.

26. Zheng K, Lu H, Sheng Z, Li Y, Xu B. Low-concentration of perifosine surprisingly protects cardiomyocytes from oxygen glucose deprivation. Biochem Biophys Res Commun. 2016; 469:753-760.

27. den Uyl D, Bultink IE, Lems WF. Advances in glucocorticoid-induced osteoporosis. Curr Rheumatol Rep. 2011; 13:233-240.

28. Weinstein RS. Clinical practice. Glucocorticoid-induced bone disease. N Engl J Med. 2011; 365:62-70.

29. Kerachian MA, Seguin C, Harvey EJ. Glucocorticoids in osteonecrosis of the femoral head: a new understanding of the mechanisms of action. J Steroid Biochem Mol Biol. 2009; 114:121-128.

30. Xu Y, Li G, Du B, Zhang P, Xiao L, Sirois P, Li K. Hepcidin increases intracellular $\mathrm{Ca} 2+$ of osteoblast hFOB1.19 through L-type Ca2+ channels. Regul Pept. 2011; 172:58-61.

31. Zhu LQ, Zhen YF, Zhang Y, Guo ZX, Dai J, Wang XD. Salinomycin activates AMP-activated protein kinasedependent autophagy in cultured osteoblastoma cells: a negative regulator against cell apoptosis. PLoS One. 2013; 8:e84175.

32. Hentges KE, Kyttala M, Justice MJ, Peltonen L. Comparative physical maps of the human and mouse Meckel syndrome critical regions. Mamm Genome. 2004; 15:252-264. 
33. Valeri N, Braconi C, Gasparini P, Murgia C, Lampis A, Paulus-Hock V, Hart JR, Ueno L, Grivennikov SI, Lovat F, Paone A, Cascione L, Sumani KM, et al. MicroRNA-135b promotes cancer progression by acting as a downstream effector of oncogenic pathways in colon cancer. Cancer Cell. 2014; 25:469-483.

34. Umezu T, Tadokoro H, Azuma K, Yoshizawa S, Ohyashiki K, Ohyashiki JH. Exosomal miR-135b shed from hypoxic multiple myeloma cells enhances angiogenesis by targeting factor-inhibiting HIF-1. Blood. 2014; 124:3748-3757.

35. Chen MB, Wei MX, Han JY, Wu XY, Li C, Wang J, Shen W, Lu PH. MicroRNA-451 regulates AMPK/mTORC1 signaling and fascin 1 expression in HT-29 colorectal cancer. Cell Signal. 2014; 26:102-109.

36. Lu PH, Chen MB, Ji C, Li WT, Wei MX, Wu MH. Aqueous Oldenlandia diffusa extracts inhibits colorectal cancer cells via activating AMP-activated protein kinase signalings. Oncotarget. 2016; 7: 45889-45900. doi: 10.18632/ oncotarget.9969.

37. Hu X, Jiang F, Bao Q, Qian H, Fang Q, Shao Z. Compound 13, an alpha1-selective small molecule activator of AMPK, potently inhibits melanoma cell proliferation. Tumour Biol. 2015. 